JOURNAL OF INTEGRAL EQUATIONS

AND APPLICATIONS

Volume 5, Number 3, Summer 1993

\title{
LOCAL EXISTENCE FOR ABSTRACT SEMILINEAR VOLTERRA INTEGRODIFFERENTIAL EQUATIONS
}

\author{
SERGIU AIZICOVICI AND KENNETH B. HANNSGEN
}

ABSTRACT. Local existence is proved for mild solutions of a linear Volterra equation of convolution type in a Banach space, perturbed by a continuous nonlinear hereditary term. The linear part, which involves an unbounded linear operator, has a resolvent kernel with certain compactness properties that permit one to use Schauder's theorem to obtain local existence for the perturbed equation without stronger conditions on the nonlinearity.

1. Introduction and statement of results. For the Volterra integrodifferential equation

$$
\begin{gathered}
\mathbf{x}^{\prime}(t)=\int_{0}^{t} a(t-\tau) \mathbf{L x}(\tau) d \tau+\mathbf{f}(t), \quad t>0 \\
\mathbf{x}(0)=\mathbf{x}_{0},
\end{gathered}
$$

where $\mathbf{L}$ is a linear operator in a Banach space $\mathcal{X}$, and $\mathbf{f} \in L_{\text {loc }}^{1}([0, \infty) ; \mathcal{X})$, a resolvent kernel is an operator-valued function $\mathbf{S}(t)$ such that the solution of (1.1) is given by

$$
\mathbf{x}(t)=\mathbf{S}(t) \mathbf{x}_{0}+\int_{0}^{t} \mathbf{S}(t-\tau) \mathbf{f}(\tau) d \tau
$$

In what follows, $a(t)$ is a real-valued kernel satisfying

$$
a \in L_{\mathrm{loc}}^{1}\left(\mathbf{R}^{+}\right) \text {is positive, nonincreasing }
$$
and convex on $(0, \infty)$ with $0<a(0+) \leq \infty$.

Here $\mathbf{R}^{+}=[0, \infty)$. The unknown $\mathbf{x}$ takes its values in a Banach space $(\mathcal{X},\|\cdot\|)$ and

$\mathbf{L}$ is a closed linear operator in $\mathcal{X}$, defined on the dense domain $\mathcal{D}$, and $\mathbf{L}$ is invertible with $\mathbf{L}^{-1}$ compact on $\mathcal{X}$.

Received by the editors on January 28, 1993

Research by the second author was partially supported by the Air Force Office of Scientific Research under grant AFOSR-91-0083.

Copyright (C)1993 Rocky Mountain Mathematics Consortium 
We shall use (1.2), together with certain compactness properties of $\mathbf{S}(t)$ and Schauder's fixed-point theorem, to prove local existence theorems for mild solutions of nonlinear perturbations of (1.1) of the form

$$
\begin{gathered}
\mathbf{x}^{\prime}(t)=\int_{0}^{t} a(t-\tau) \mathbf{L x}(\tau) d \tau+(\mathbf{F x})(t), \quad 0<t<T \\
\mathbf{x}(0)=\mathbf{x}_{0},
\end{gathered}
$$

where $\mathbf{F}$ is a continuous hereditary mapping defined on the space $\mathcal{C}_{T}:=C([0, T] ; \mathcal{O})$, and where $T>0$, the open subset $\mathcal{O}$ of $\mathcal{X}$, and the initial value $\mathbf{x}_{0} \in \mathcal{O}$ are considered fixed throughout the paper. More specifically, we assume that there exists a $p, 1 \leq p \leq \infty$, such that

(i) $\mathbf{F}: \mathcal{C}_{T} \rightarrow L^{p}(0, T ; \mathcal{X})$ is continuous

(ii) for $0<T^{\prime}<T$, the formula $\mathbf{F}_{T^{\prime}}\left(\left.\mathbf{h}\right|_{T^{\prime}}\right)=\left.\mathbf{F}(\mathbf{h})\right|_{T^{\prime}}$ gives a (well defined) continuous map from $\mathcal{C}_{T^{\prime}}$ to $L^{p}\left(0, T^{\prime} ; \mathcal{X}\right)$.

(Here $\left.\right|_{T^{\prime}}$ denotes restriction to $\left[0, T^{\prime}\right]$. Lebesgue integral notation refers to the Bochner integral [10].) For example, we could have $p=\infty, \mathcal{O}=\mathcal{X}$ and

$$
(\mathbf{F h})(t)=\mathbf{g}_{1}(t, \mathbf{h}(t))+\int_{0}^{t} b(t-\tau) \mathbf{g}_{2}(\tau, \mathbf{h}(\tau)) d \tau
$$

with $\mathbf{g}_{1}$ and $\mathbf{g}_{2}$ continuous from $[0, T] \times \mathcal{X}$ to $\mathcal{X}$ and $b \in L^{1}(0, T ; \mathbf{R})$.

A mild solution of $(1.5)$ on $[0, T]$ is a function $\mathbf{x} \in \mathcal{C}_{T}$ that satisfies

$$
\mathbf{x}(t)=\mathbf{x}_{0}+\mathbf{L} \int_{0}^{t} A(t-\tau) \mathbf{x}(\tau) d \tau+\int_{0}^{t}(\mathbf{F x})(\tau) d \tau
$$

on the interval, with $A(t)=\int_{0}^{t} a(\tau) d \tau$. 
The properties of $\mathbf{S}(t)$ that we shall need can be established in two (overlapping) settings:

(i) $-a^{\prime}$ is convex, $\mathcal{X}$ is a separable Hilbert space

and $\mathbf{L}$ is self-adjoint and strictly negative definite,

or

(ii) $a$ is log-convex, with $a^{\prime}$ locally absolutely continuous on $(0, \infty)$ in the case where $a(0+)<\infty$, and $\mathbf{L}$ generates a strongly continuous cosine family on $\mathcal{X}$.

Remark. A function $a(t)$ on $(0, T]$ that satisfies our hypotheses (convexity, etc.) on that interval can be extended to all of $(0, \infty)$ in such a way that the hypotheses still hold. To enable us to use certain results concerning $\mathbf{S}(t)$, established via transform arguments, we assume that this extension has already been made.

Our first result relies on compactness of $\mathbf{S}(t)$, and the essential condition for this is

$$
a^{\prime}(0+)=-\infty .
$$

Theorem 1.1. Let (1.3), (1.4), (1.6) with $p>1$, (1.9) (either (i) or (ii)) and (1.10) hold. Then there exists $T^{\prime}, 0<T^{\prime} \leq T$, such that (1.5) has a mild solution on $\left[0, T^{\prime}\right]$.

Our second result concerns equations (1.5) of the special form

$$
\begin{gathered}
\mathbf{x}^{\prime}(t)=\int_{0}^{t} a(t-\tau)[\mathbf{L x}(\tau)+\mathbf{g}(\tau, \mathbf{x}(\tau))] d \tau+\mathbf{f}(t), \quad t>0 \\
\mathbf{x}(0)=\mathbf{x}_{0} ;
\end{gathered}
$$

in other words, we consider (1.5) with

$$
(\mathbf{F x})(t)=\int_{0}^{t} a(t-\tau) \mathbf{g}(\tau, \mathbf{x}(\tau)) d \tau+\mathbf{f}(t)
$$


where $\mathbf{g}:(0, T) \times \mathcal{O} \rightarrow \mathcal{X}$ is given and

$$
\mathbf{f} \in L^{1}(0, T ; \mathcal{X})
$$

Using the resolvent identity

$$
\mathbf{S}^{\prime}(t) \mathbf{L}^{-1} \mathbf{x}=\int_{0}^{t} a(t-\tau) \mathbf{S}(\tau) \mathbf{x} d \tau, \quad \mathbf{x} \in \mathcal{X}
$$

(see (2.1) below) in (1.2), with $(\mathbf{F x})+\mathbf{f}$ in place of $\mathbf{f}$, we see that the mild version of (1.11) is formally equivalent to

$$
\mathbf{x}(t)=\mathbf{S}(t) \mathbf{x}_{0}+\int_{0}^{t} \mathbf{S}^{\prime}(t-\tau) \mathbf{L}^{-1} \mathbf{g}(\tau, \mathbf{x}(\tau)) d \tau+\int_{0}^{t} \mathbf{S}(t-\tau) \mathbf{f}(\tau) d \tau
$$

When (1.10) holds we shall prove local existence for (1.11) by extending the argument for Theorem 1.1 to the case $p=1$ for $\mathbf{F}$ as in (1.12). If (1.10) does not hold, then necessarily (cf. (1.3))

$$
a(0+)<\infty,
$$

and this will imply the required compactness property of $\mathbf{S}^{\prime}(t) \mathbf{L}^{-1}$ in (1.15). Concerning the nonlinearity, we assume

$$
g:(0, T) \times \mathcal{O} \rightarrow \mathcal{X} \text { satisfies }
$$

(i) $\mathbf{g}(t, \mathbf{x})$ is measurable in $t$ for each $\mathbf{x} \in \mathcal{O}$,

(ii) $\mathbf{g}(t, \mathbf{x})$ is continuous in $\mathbf{x}$, for a.e. $t \in(0, T)$,

(iii) there exist a closed ball $\mathcal{B}$ centered at $\mathbf{x}_{0}$, with $\mathcal{B} \subset \mathcal{O}$ and a function $\psi \in L^{1}\left(0, T ; \mathbf{R}^{+}\right)$such that $\|\mathbf{g}(t, \mathbf{x})\| \leq \psi(t)$ a.e. on $(0, T)$ for all $\mathbf{x} \in \mathcal{B}$.

In particular, then, $\mathbf{F}$ given by (1.12) satisfies (1.6) with $p=1$ (in a ball centered at $\mathbf{x}_{0}$.)

Theorem 1.2. Let (1.3), (1.4), (1.9) (either (i) or (ii)), (1.13) and (1.17) hold. Then there exists $T^{\prime} \in(0, T]$ such that (1.11) has a mild solution on $\left[0, T^{\prime}\right]$. 
Remarks. The substitution $\mathbf{L}_{k}=\mathbf{L}-k \mathbf{I}(k \in \mathbf{R})$ transforms (1.5) into

$$
\mathbf{x}^{\prime}=a * \mathbf{L}_{k} \mathbf{x}+\mathbf{F}_{k} \mathbf{x}
$$

with $\mathbf{F}_{k} \mathbf{x}=\mathbf{F} \mathbf{x}+k a * \mathbf{x}$. Thus our results remain valid if we merely require (1.4) to hold for some $\mathbf{L}_{k}$.

There is a vast literature on local existence of solutions to classes of differential and integral equations involving discontinuous nonlinearities, and particularly those involving nonlinear differential operators. There are, in addition, some results where the nonlinearity is continuous, as in this paper. For the problem

$$
\mathbf{u}^{\prime}(t)=\mathbf{A u}(t)+\mathbf{f}(t, \mathbf{u}(t))
$$

where $\mathbf{A}$ generates a compact $C_{0}$-semigroup $\mathbf{T}(t)$ in $\mathcal{X}$ and $\mathbf{f}$ is continuous, see $[\mathbf{1 2}, \mathbf{1 3}]$, as well as the compactness results for the mapping $\mathbf{g} \rightarrow \mathbf{T} * \mathbf{g}$ given in [3]. For earlier work on the specific case of (1.11) with $\mathbf{L}=\triangle$ and $\mathcal{X}=L^{2}(\Omega)$ on a bounded domain $\Omega$ in $\mathbf{R}^{n}\left(a \in W_{\text {loc }}^{2,1}\left(\mathbf{R}^{+}\right), a(0)>0\right)$, and with a sign condition on the continuous nonlinearity, see [1]. An integrated version of (1.11) falls within the scope of $[\mathbf{2}, \mathbf{9}]$; however, the respective results are not applicable to (1.11), due to restrictions on the convolution kernel.

The plan of the current paper is as follows. In Section 2 we discuss $\mathbf{S}(t)$ and prove a general existence result, based on the Schauder fixedpoint theorem, that identifies the compactness property (2.4) needed for the proofs of Theorems 1.1 and 1.2. These proofs, including two compactness lemmas of independent interest, appear in Section 3. Finally, in Section 4, we give an extension of Theorem 1.2 to cover multi-valued nonlinearities $\mathbf{g}$.

2. Resolvent kernels and a general existence theorem. In this section we collect some facts concerning $\mathbf{S}(t)$, and we use the Schauder fixed-point theorem to prove a general result that reduces the proofs of Theorems 1.1 and 1.2 to an examination of $\mathbf{S}(t)$ and $\mathbf{S}^{\prime}(t) \mathbf{L}^{-1}$ respectively.

Throughout the paper, we write $\|\cdot\|$ for the norm in $\mathcal{X}$, and also for the uniform operator norm in $\mathcal{L}(\mathcal{X})=\mathcal{L}(\mathcal{X}, \mathcal{X})$, the space of 
bounded linear operators in $\mathcal{X}$. When $\mathcal{X}$ is a Hilbert space, its inner product is denoted $\langle\cdot, \cdot\rangle$. We let $\mathcal{X}_{1}$ denote the Banach space $\left(\mathcal{D},\|\cdot\|_{1}\right)$, with $\|\mathbf{x}\|_{1}=\|\mathbf{L x}\|+\|\mathbf{x}\|$. Convolutions will sometimes be written $f * g(t)=\int_{0}^{t} f(t-\tau) g(\tau) d \tau$.

We follow Prüss [14-16] for much of the discussion of equations and resolvents in $\mathcal{X}$. For comparison, note that we always have $A(0)=0$ and that $a(t)=A^{\prime}(t)$ here corresponds to $a_{\infty}+a_{1}(t)$, e.g. in $[\mathbf{1 6}$, Equation (1.1)].

The hypotheses of Theorems 1.1 and 1.2 ensure that (1.1) has a resolvent $\mathbf{S}(t)$ in the sense of [16, Definition 1.3]; thus

(i) $\{\mathbf{S}(t): t \geq 0\}$ is a strongly continuous family of bounded linear operators on $\mathcal{X}$ with $\mathbf{S}(0)=\mathbf{I}$,

(ii) $\mathbf{S}(t)$ commutes with $\mathbf{L}$ and satisfies the resolvent identity $\mathbf{S}(t) \mathbf{x}=\mathbf{x}+(A * \mathbf{L S}(\cdot) \mathbf{x})(t), t \geq 0, \mathbf{x} \in \mathcal{X}_{1}$.

Note that (1.14) follows from the identity in (2.1) (ii). In addition, (2.1)(i) and the uniform boundedness principle imply that

$$
\sigma:=\sup \{\|\mathbf{S}(t)\|: 0 \leq t \leq T\}<\infty
$$

To define $\mathbf{S}(t)$ when (1.9)(i) holds $[\mathbf{4}, \mathbf{5}]$, recall that, since $\mathbf{L}^{-1}$ is compact and self-adjoint, $\mathbf{L}$ has the spectral decomposition

$$
\mathbf{L}=\int_{\mathbf{R}} \lambda d \mathbf{E}_{\lambda}=\sum_{k=1}^{\infty} \lambda_{k}<\cdot, \mathbf{y}_{k}>\mathbf{y}_{k},
$$

where $0>\lambda_{1} \geq \lambda_{2} \geq \cdots \rightarrow-\infty$ are the eigenvalues of $\mathbf{L}$ and $\left\{\mathbf{y}_{k}\right\}$ is an orthonormal eigenvector basis. Then

$$
\mathbf{S}(t) \mathbf{x}=\sum_{k=1}^{\infty} u_{k}(t)<\mathbf{x}, \mathbf{y}_{k}>\mathbf{y}_{k}
$$

where $u_{k}$ satisfies the scalar equation

$$
u_{k}(t)=1+\lambda_{k}\left(A * u_{k}\right)(t)
$$


Condition (1.9)(ii) leads to $\mathbf{S}(t)$ through the general existence theorem of Da Prato and Iannelli [6] and the estimates of [14].

For our purposes, the map

$$
\mathbf{\Phi}: L^{p}(0, T ; \mathcal{X}) \rightarrow C([0, T] ; \mathcal{X})
$$

$(1 \leq p \leq \infty)$ defined by

$$
\Phi \mathbf{h}(t)=\mathbf{S} * \mathbf{h}(t)
$$

is central. Since $\mathbf{S}(t)$ is strongly continuous, an argument involving the dominated convergence theorem shows that $\Phi \mathbf{h}$ does indeed belong to $C([0, T] ; \mathcal{X})$, and an easy estimate establishes that $\Phi$ is continuous. From [16, Proposition 1.2ii] we have

Theorem 2.1. Assume (1.3), (1.4) and (1.6). Then each fixed point in $\mathcal{C}_{T}$ of the map $\Phi_{1}$ given by

$$
\Phi_{1} \mathbf{h}=\mathbf{S}(\cdot) \mathbf{x}_{0}+\Phi \circ \mathbf{F h}
$$

is a mild solution of (1.5).

We have, then, the following general criterion for local existence.

Theorem 2.2. Let (1.3), (1.4), (1.6) and (2.1) be satisfied. In addition, assume

there exists $\rho>0$, with

$\mathcal{B}=\mathcal{B}_{\rho}\left(\mathbf{x}_{0}\right):=\left\{\mathbf{x} \in \mathcal{X}:\left\|\mathbf{x}-\mathbf{x}_{0}\right\| \leq \rho\right\} \subset \mathcal{O}$

such that $\{\Phi \circ \mathbf{F}(\mathbf{h}): \mathbf{h} \in C([0, T] ; \mathcal{B})\}$

is precompact in $C([0, T] ; \mathcal{X})$.

Then there exists $T^{\prime}, 0<T^{\prime} \leq T$, such that (1.5) has a mild solution on $\left[0, T^{\prime}\right]$.

So we reduce the proofs of Theorems 1.1 and 1.2 to establishing (2.4). 
Proof of Theorem 2.2. By (1.6), we can choose $M>0$ and $\rho$ in (2.4) in such a way that

$$
\|\mathbf{F}(\mathbf{h})\|_{L^{p}(0, T ; \mathcal{X})} \leq M \quad \text { if } \mathbf{h} \in C([0, T] ; \mathcal{B}) .
$$

Then choose $T^{\prime} \in(0, T]$ such that

$$
\left\|\mathbf{S}(t) \mathbf{x}_{0}-\mathbf{x}_{0}\right\|+\sigma M\left(T^{\prime}\right)^{1 / q} \leq \rho, \quad 0 \leq t \leq T^{\prime},
$$

where $p^{-1}+q^{-1}=1$. Now let $\mathcal{Z}=C\left(\left[0, T^{\prime}\right] ; \mathcal{B}\right)$. Clearly, $\mathcal{Z}$ is a bounded, closed, convex subset of $\mathcal{C}_{T^{\prime}}$. By (2.5) and (2.6), the map $\Phi_{\mathbf{1}}$ restricts to a continuous self map of $\mathcal{Z}$. Moreover, by (1.6) and (2.3), $\left.(\Phi \circ \mathbf{F})\right|_{\mathcal{Z}}$ inherits the compactness property (2.4), and so the image of $\mathcal{Z}$ under $\Phi_{1}$ is precompact. Our claim now follows from Schauder's fixed-point theorem and Theorem 2.1.

3. Proofs of Theorems 1.1 and 1.2. We must show that the hypotheses of the two theorems imply (2.4). For Theorem 1.1 and the case of Theorem 1.2 where (1.10) holds, the main step is given by the following general result.

Lemma 3.1. Let the conditions (1.3), (1.4), (1.9) (either (i) or (ii)) and $(1.10)$ be satisfied. Let $\Phi: L^{p}(0, T ; \mathcal{X}) \rightarrow C([0, T] ; \mathcal{X})$ be defined by $(2.3), 1 \leq p \leq \infty$. Then $\Phi$ maps every uniformly integrable subset of $L^{1}(0, T ; \mathcal{X})$ into a precompact subset of $C([0, T] ; \mathcal{X})$. In particular, if $p>1$, then $\Phi$ is a compact operator.

Proof of Lemma 3.1. Let $\mathcal{H} \subset L^{1}=L^{1}(0, T ; \mathcal{X})$ be uniformly integrable, i.e., $\int_{E}\|\mathbf{h}(t)\| d t \rightarrow 0$ as $m(E) \rightarrow 0$ (for all measurable $E, m=$ Lebesgue measure), uniformly for $\mathbf{h} \in \mathcal{H}$. Then there exists $r<\infty$ with $\|\mathbf{h}\|_{L^{1}}<r, \mathbf{h} \in \mathcal{H}$. We shall show that $\Phi(\mathcal{H})$ is equicontinuous at each $t$ in $[0, T]$ and that $\Phi(\mathcal{H})(t)=\{(\Phi \mathbf{h})(t): \mathbf{h} \in \mathcal{H}\}$ is precompact in $\mathcal{X}(0 \leq t \leq T)$. By the Arzèla-Ascoli theorem, this will establish that $\Phi(\mathcal{H})$ is precompact in $C([0, T] ; \mathcal{X})$. The final assertion of the Lemma follows because balls in $L^{p}, p>1$, are uniformly integrable subsets of $L^{1}$ :

$$
\int_{E}|\psi| d t \leq m(E)^{1 / q}\|\psi\|_{p}, \quad 1 / q+1 / p=1 .
$$


We know that $\|\mathbf{S}(t)\| \leq \sigma, 0 \leq t \leq T$. Under our hypotheses, moreover, $\mathbf{S}(t)$ is continuous on $(0, T]$ in the uniform operator topology. This fact is given in [14, Theorem 7II(i)] for the case where (1.9)(ii) holds. Under hypothesis (1.9)(i), it is a consequence of (2.2) and

$$
u_{k}(t) \rightarrow 0(k \rightarrow \infty), \quad \text { uniformly for } 0<t_{0} \leq t<\infty
$$

(cf. [8, Theorem 1]), since

$$
\| \mathbf{S}\left(t_{1}\right)-\mathbf{S}\left(t_{2}\right)||=\sup _{k}\left|u_{k}\left(t_{1}\right)-u_{k}\left(t_{2}\right)\right|
$$

and each $u_{k}$ is continuous. (In the theorems from [8] and [14] cited here, (1.10) is used.)

Let $0<t_{0}<T$. For $t_{0} \leq t_{1} \leq t_{2} \leq T, \mathbf{h} \in \mathcal{H}, 0<\mu<t_{0} / 2$, we have

$$
\begin{aligned}
\left\|(\Phi \mathbf{h})\left(t_{2}\right)-(\Phi \mathbf{h})\left(t_{1}\right)\right\| \leq & \int_{0}^{t_{1}-\mu}\left\|\mathbf{S}\left(t_{2}-\tau\right)-\mathbf{S}\left(t_{1}-\tau\right)\right\|\|\mathbf{h}(\tau)\| d \tau \\
& +\int_{t_{1}-\mu}^{t_{1}}\left\|\mathbf{S}\left(t_{2}-\tau\right)-\mathbf{S}\left(t_{1}-\tau\right)\right\|\|\mathbf{h}(\tau)\| d \tau \\
& +\int_{t_{1}}^{t_{2}}\left\|\mathbf{S}\left(t_{2}-\tau\right)\right\|\|\mathbf{h}(\tau)\| d \tau \\
\leq & \max _{\mu \leq s \leq t_{1}}\left\|\mathbf{S}\left(s+t_{2}-t_{1}\right)-\mathbf{S}(s)\right\| r \\
& +2 \sigma \int_{t_{1}-\mu}^{t_{2}}\|\mathbf{h}(t)\| d t
\end{aligned}
$$

Given $\epsilon>0$, we can choose $\mu$ and then $\delta>0$ so that the last expression is less than $\epsilon$ whenever $t_{0} \leq t_{1} \leq t_{2} \leq t_{1}+\delta$, uniformly for $\mathbf{h} \in \mathcal{H}$. This gives us equicontinuity at any $t \in(0, T]$, and the argument for $t=0$ is similar.

Precompactness of $\Phi(\mathcal{H})(0)=\{0\}$ is trivial. For $0<t \leq T$ we have $(3.2)$

$$
\begin{aligned}
\left\|\Phi \mathbf{h}(t)-\int_{\delta}^{t} \mathbf{S}(\tau) \mathbf{h}(t-\tau) d \tau\right\| & =\left\|\int_{0}^{\delta} \mathbf{S}(\tau) \mathbf{h}(t-\tau) d \tau\right\| \\
& \leq \sigma \sup _{\mathbf{h} \in \mathcal{H}}\left\{\int_{t-\delta}^{t}\|\mathbf{h}(t)\| d t\right\}, \quad 0<\delta<t .
\end{aligned}
$$


Given $\epsilon>0$, we shall find an operator $\mathbf{S}_{\epsilon}(\tau)=\mathbf{Q}_{\epsilon} \mathbf{T}_{\epsilon}(\tau)$ with $\mathbf{Q}_{\epsilon}$ compact, $\mathbf{T}_{\epsilon}(\tau)$ uniformly bounded and $\left\|\mathbf{S}(\tau)-\mathbf{S}_{\epsilon}(\tau)\right\|<\epsilon, \delta \leq \tau \leq t$. Then

$$
\left\|\int_{\delta}^{t} \mathbf{S}(\tau) \mathbf{h}(t-\tau) d \tau-\mathbf{Q}_{\epsilon} \int_{\delta}^{t} \mathbf{T}_{\epsilon}(\tau) \mathbf{h}(t-\tau) d \tau\right\| \leq \epsilon r .
$$

On the other hand,

$$
\left\|\int_{\delta}^{t} \mathbf{T}_{\epsilon}(\tau) \mathbf{h}(t-\tau) d \tau\right\| \leq \sup _{\delta \leq \tau \leq t}\left\|\mathbf{T}_{\epsilon}(\tau)\right\| r
$$

so $\left\{\mathbf{Q}_{\epsilon} \int_{\delta}^{t} \mathbf{T}_{\epsilon}(\tau) \mathbf{h}(t-\tau) d \tau: \mathbf{h} \in \mathcal{H}\right\}$ is contained in a fixed compact $\mathcal{K}=\mathcal{K}(\delta, \epsilon)$ of $\mathcal{X}$ with dist $(\mathcal{K}, \Phi(\mathcal{H})(t)) \rightarrow 0$ as $\delta, \epsilon \rightarrow 0$ (cf. $(3.2))$. Thus $\Phi(\mathcal{H})(t)$ is totally bounded and therefore precompact.

For the construction of $\mathbf{S}_{\epsilon}(\tau)$, we take the two cases of (1.9) separately. For (1.9)(i), return to (2.2) and recall (3.1). Let

$$
\mathbf{P}_{N} \mathbf{x}=\sum_{k=1}^{N}<\mathbf{x}, \mathbf{y}_{k}>\mathbf{y}_{k}
$$

and $\mathbf{T}_{\epsilon}(\tau)=\mathbf{S}(\tau)$. Then

$$
\left\|\mathbf{P}_{N} \mathbf{T}_{\epsilon}(\tau)-\mathbf{S}(\tau)\right\|=\sup _{k>N}\left|u_{k}(\tau)\right| \rightarrow 0, \quad N \rightarrow \infty,
$$

uniformly for $\delta \leq \tau \leq T,\left\{\mathbf{T}_{\epsilon}(\tau)\right\}$ is uniformly bounded, and $\mathbf{P}_{N}$ is compact. We take $\mathbf{Q}_{\epsilon}=\mathbf{P}_{N}$ with $N=N(\epsilon, \delta)$ sufficiently large.

Finally, assume (1.9)(ii). Since $\mathbf{S}(t)$ is continuous on $(0, \infty)$ in the uniform operator topology, we know from [10, Theorem 6.3.2] that $\mathbf{S}(t)=\lim _{N \rightarrow \infty} \mathbf{S}_{N, \gamma}(t)$, with

$$
\mathbf{S}_{N, \gamma}(t)=\frac{e^{\gamma t}}{2 \pi} \int_{-N}^{N}\left(1-\frac{|\omega|}{N}\right) e^{i \omega t} \hat{\mathbf{S}}(\gamma+i \omega) d \omega
$$

for sufficiently large $\gamma$, uniformly for $\delta \leq t \leq T$. Now by [14], $\hat{\mathbf{S}}(\lambda)=-\mathbf{L}^{-1} \mathbf{R}(\lambda)$, where

$$
\mathbf{R}(\lambda)=\left[\mathbf{I}-\frac{\lambda}{\hat{a}(\lambda)}\left(\frac{\lambda}{\hat{a}(\lambda)} \mathbf{I}-\mathbf{L}\right)^{-1}\right] / \hat{a}(\lambda) .
$$


We set $\mathbf{S}_{\epsilon}=\mathbf{S}_{N, \gamma}, \mathbf{Q}_{\epsilon}=-\mathbf{L}^{-1}$,

$$
\mathbf{T}_{\epsilon}(t)=\frac{e^{\gamma t}}{2 \pi} \int_{-N}^{N}\left(1-\frac{|\omega|}{N}\right) e^{i \omega t} \mathbf{R}(\gamma+i \omega) d \omega
$$

with $N=N(\epsilon, \sigma)$ sufficiently large and get the required decomposition. This completes the proof of Lemma 3.1.

Theorem 1.1 now follows immediately since by (1.6), there is a ball $\mathcal{B}$ as in $(2.4)$ such that $\mathbf{F}$ maps $\mathcal{B}$ into a bounded subset of $L^{p}(0, T ; \mathcal{X})$.

For Theorem 1.2, if (1.10) holds, we can again establish (2.4), with $\mathcal{B}$ given by (1.17) (iii), since $\mathbf{F}\left(\mathcal{C}_{T}\right)$ is then uniformly integrable.

When (1.10) fails, we must have (1.16). We write $\Phi \circ \mathbf{F}(\mathbf{h})=$

$\Phi\left(a * \mathbf{G}_{\mathbf{h}}+\mathbf{f}\right)$ with $\mathbf{G}_{\mathbf{h}}(t)=\mathbf{g}(t, \mathbf{h}(t))$. By (1.17), $\left\{\mathbf{G}_{\mathbf{h}}: \mathbf{h} \in C([0, T] ; \mathcal{B})\right\}$ is a bounded set in $L^{1}(0, T ; \mathcal{X})$. Then $(2.4)$, and hence the remaining part of Theorem 2.1, is a consequence of the following general result.

Lemma 3.2. Let (1.3), (1.4), (1.9) (either (i) or (ii)) and (1.16) hold. Then the mapping $\mathbf{G} \rightarrow \Phi(a * \mathbf{G})=\mathbf{S} * a * \mathbf{G}$ from $L^{1}(0, T ; \mathcal{X})$ to $C([0, T] ; \mathcal{X})$ is compact.

Proof of Lemma 3.2. By (1.14),

$$
\Phi(\mathbf{a} * \mathbf{G})=\mathbf{S}^{\prime} \mathbf{L}^{-1} * \mathbf{G} .
$$

Let $\mathbf{C}(t)$ be the cosine family generated by $\mathbf{L}$ and define the Banach space

$$
\mathcal{Y}=\left\{\mathbf{x} \in \mathcal{X}: \mathbf{C}(\cdot) \mathbf{x} \in C^{1}(\mathbf{R} ; \mathcal{X})\right\}
$$

with norm $\|\mathbf{x}\| \mathcal{Y}=\|\mathbf{x}\|+\sup \left\{\left\|\mathbf{C}^{\prime}(t) \mathbf{x}\right\|: 0 \leq t \leq 1\right\}$. In the Hilbert space case $(1.9)(\mathrm{i}), \mathcal{Y}$ can be identified with the domain of $(-\mathbf{L})^{1 / 2}$ with the graph norm (see [17, Proposition 3.4]), and it is clear that the injection $\mathbf{J}: \mathcal{Y} \rightarrow \mathcal{X}$ is compact. The same thing is true if (1.9)(ii) holds: we can approximate $\mathbf{J}$ in $\mathcal{L}(\mathcal{Y}, \mathcal{X})$ with the operators $t^{-1} \mathbf{L}^{-1} \mathbf{C}^{\prime}(t)=t^{-1} \int_{0}^{t} \mathbf{C}(\tau) d \tau, t \rightarrow 0+$, and these are compact because $\mathbf{C}^{\prime}(t) \in \mathcal{L}(\mathcal{Y}, \mathcal{X})$ and $\mathbf{L}^{-1}$ is compact. 
By [5, Theorem 3.1 ii], $\mathbf{S}^{\prime}(\cdot) \mathbf{L}^{-1}: \mathbf{R}^{+} \rightarrow \mathcal{L}(\mathcal{X}, \mathcal{Y})$ is strongly continuous when (1.9)(i) holds. If (1.9)(ii) holds, the same is true by [15, Equation (3.7)] (see Remark below). Then $\mathbf{S}^{\prime} \mathbf{L}^{-1} * \mathbf{G}$ exists in $\mathcal{Y}$, and by (3.3),

$$
\sup _{0 \leq t \leq T}\|\Phi(a * \mathbf{G})(t)\| \mathcal{Y} \leq \sigma^{\prime}\|\mathbf{G}\|_{L^{1}(0, T ; \mathcal{X})}
$$

where $\sigma^{\prime}=\sup \left\{\left\|\mathbf{S}^{\prime}(t) \mathbf{L}^{-1}\right\|_{\mathcal{L}(\mathcal{X}, \mathcal{Y})}: 0 \leq t \leq T\right\}<\infty$. In particular, if $\mathcal{G}$ is a bounded subset of $L^{1}(0, T ; \mathcal{X})$, then $\{\Phi(a * \mathbf{G})(t): \mathbf{G} \in \mathcal{G}\}$ is precompact in $\mathcal{X}$. Since $\mathbf{S}(t)$ is uniformly bounded, standard estimates show that $\{\Phi(a * \mathbf{G}): \mathbf{G} \in \mathcal{G})\}$ is equicontinuous at each point of $[0, T]$. This proves Lemma 2.2 and completes the proof of Theorem 1.2.

Remark. For strong continuity of $\mathbf{S}^{\prime}(\cdot) \mathbf{L}^{-1}$ when (1.9)(ii) holds, one can use the representation

$$
\hat{\mathbf{S}}^{\prime}(\lambda)=\alpha(\lambda) \int_{0}^{\infty} \mathbf{C}^{\prime}(\tau) \frac{\beta(\lambda)}{\lambda} e^{-\beta(\lambda) \tau} d \tau
$$

with $\alpha(\lambda)=\left(a(0+)+\hat{a}^{\prime}(\lambda)\right)^{1 / 2}, \beta(\lambda)=\lambda / \alpha(\lambda)$ (cf. [15, Equations (2.6), $(3.7)])$. A transform argument based on the Wiener-Lévy theorem shows that $\alpha(\lambda)-a(0+)^{1 / 2}$ is the Laplace transform of a locally integrable function $b$ on $\mathbf{R}^{+}$, while the arguments of [14, Section 4], together with the standard estimate

$$
\left\|\mathbf{C}^{\prime}(\tau)\right\|_{\mathcal{L}(\mathcal{X}, \mathcal{Y})} \leq K e^{\omega t}
$$

(for some $K, \omega>0$ ), show that the integral factor in (3.4) is the transform of a strongly continuous function $\mathbf{T}: \mathbf{R}^{+} \rightarrow \mathcal{L}(\mathcal{X}, \mathcal{Y})$. Now $\mathbf{S}^{\prime}(t) \mathbf{L}^{-1}=\left(a(0+)^{1 / 2} \mathbf{T}(t)+(b * \mathbf{T})(t)\right) \mathbf{L}^{-1}$ and we are done.

4. A multivalued nonlinearity. We outline here an extension of Theorem 1.2 to the case of multivalued nonlinearities. The approach is an adaptation of that in [2]; see [18, Chapter 3] for a discussion of multivalued perturbations for abstract differential equations and [19] for preliminaries on multivalued mappings.

Throughout this section we assume that $\mathcal{X}$ is separable. Consider the Volterra integrodifferential inclusion (cf. (1.11))

$$
\begin{gathered}
\mathbf{x}^{\prime}(t)-\int_{0}^{t} a(t-\tau)[\mathbf{L x}(\tau)+\mathbf{G}(\tau, \mathbf{x}(\tau))] d \tau \ni \mathbf{f}(t), \quad 0<t<T \\
\mathbf{x}(0)=\mathbf{x}_{0},
\end{gathered}
$$


where (1.3), (1.4), (1.9) ((i) or (ii)) and (1.13) hold, $\mathbf{x}_{0} \in \mathcal{X}$, and $\mathbf{G}:[0, T] \times \mathcal{B} \rightarrow 2^{\mathcal{X}} \backslash\{\phi\}\left(\mathcal{B}=\mathcal{B}_{\rho}\left(\mathbf{x}_{0}\right), \rho>0\right)$, is a multivalued perturbation satisfying

(i) $\mathbf{G}$ is closed valued and measurable,

(ii) $\mathbf{x} \rightarrow \mathbf{G}(t, \mathbf{x})$ is lower semicontinuous (l.s.c.), for a.e. $t \in(0, T)$,

(iii) there exists $\phi \in L^{1}\left(0, T ; \mathbf{R}^{+}\right)$such that

$$
\sup _{\mathbf{x} \in \mathcal{B}}\left(\sup _{\mathbf{y} \in \mathbf{G}(t, \mathbf{x})}\|\mathbf{y}\|\right) \leq \phi(t) \text { a.e. on }(0, T) \text {. }
$$

By a mild solution to $(4.1)$ on $[0, T]$ we mean a function $\mathbf{x} \in C([0, T] ; \mathcal{B})$ satisfying

$$
\mathbf{x}(t)=\mathbf{x}_{0}+\mathbf{L} \int_{0}^{t} A(t-\tau) \mathbf{x}(\tau) d \tau+\int_{0}^{t} A(t-\tau) \mathbf{g}(\tau) d \tau+\int_{0}^{t} \mathbf{f}(\tau) d \tau
$$

for some $\mathbf{g} \in L^{1}(0, T ; \mathcal{X})$ with $\mathbf{g}(t) \in \mathbf{G}(t, \mathbf{x}(t))$, a.e. on $(0, T)$.

Theorem 4.1. Let (1.3), (1.4), (1.9) (either (i) or (ii)), (1.13) and (4.2) hold. Then there is a $T^{\prime}$ in $(0, T]$ such that (4.1) has a mild solution on $\left[0, T^{\prime}\right]$.

Proof (Outline). As in Sections 2 and 3, we find $T^{\prime} \in(0, T]$ such that the map $\Phi_{\mathbf{1}} \mathbf{h}$ defined by

$$
\Phi_{\mathbf{1}} \mathbf{h}(t)=\mathbf{S}(t) \mathbf{x}_{0}+\mathbf{S} * \mathbf{f}(t)+\mathbf{S} * a * \mathbf{h}(t)
$$

takes the set $\mathcal{K}=\left\{\mathbf{h} \in L^{1}\left(0, T^{\prime} ; \mathcal{X}\right):\|\mathbf{h}(t)\| \leq \phi(t)\right.$ a.e. $\}$ into a compact subset $\mathcal{F}$ of $C\left(\left[0, T^{\prime}\right] ; \mathcal{B}\right)$. Let $\Psi$ be the map defined on $\mathcal{F}$ by

$$
\Psi(\mathbf{y})=\left\{\mathbf{g} \in \mathrm{E}^{1}\left(0, T^{\prime} ; \mathcal{X}\right): \mathbf{g}(t) \in \mathbf{G}(t, \mathbf{y}(t)) \text { a.e. }\right\},
$$

and taking its values in the space $\mathcal{P}$ of all closed subsets of $L^{1}\left(0, T^{\prime} ; \mathbf{X}\right)$. It is easily seen that (4.4) makes sense. By [11, Theorem 4.1], it follows that $\Psi$ is l.s.c., and it is obvious that $\Psi$ is decomposable. Applying Fryszkowski's selection theorem [7], we find a continuous, single-valued map $\boldsymbol{\eta}: \mathcal{F} \rightarrow L^{1}\left(0, T^{\prime} ; \mathcal{X}\right)$ such that $\boldsymbol{\eta}(\mathbf{y}) \in \Psi(\mathbf{y})$, for all $\mathbf{y}$ in $\mathcal{F}$. By (4.4), we then have

$$
\boldsymbol{\eta}(\mathbf{y})(t) \in \mathbf{G}(t, \mathbf{y}(t)), \text { a.e., }
$$


so that

$$
\boldsymbol{\eta}(\mathcal{F}) \subset \mathcal{K}
$$

On account of the continuity of $\boldsymbol{\eta}$ and Mazur's theorem, we also deduce that $\mathcal{Q}=\overline{\operatorname{conv}} \boldsymbol{\eta}(\mathcal{F})$ is a compact, convex subset of $L^{1}\left(0, T^{\prime} ; \mathcal{X}\right)$. By (4.6), it follows that $\mathcal{Q} \subset \mathcal{K}$, since the latter set is closed and convex in $L^{1}\left(0, T^{\prime} ; \mathcal{X}\right)$. Now define $\gamma: \mathcal{Q} \rightarrow L^{1}\left(0, T^{\prime}, \mathcal{X}\right)$ by

$$
\gamma(\mathbf{h})=\boldsymbol{\eta}\left(\Phi_{\mathbf{1}} \mathbf{h}\right), \quad \mathbf{h} \in \mathcal{Q} .
$$

We see that $\gamma$ is a continuous self-map of $\mathcal{Q}$. Applying Schauder's fixed-point theorem we now conclude that $\gamma$ has a fixed point $\mathbf{g} \in \mathcal{Q}$. By (4.5) and (4.7) this means we have found $\mathrm{g} \in L^{1}\left(0, T^{\prime} ; \mathcal{X}\right)$ with $\mathbf{g}(t) \in \mathbf{G}\left(\mathbf{t}, \Phi_{1} \mathbf{g}(t)\right)$ a.e. As in Theorem 2.1, $\mathbf{y}=\Phi_{1} \mathbf{g}$ is the desired mild solution of (4.1) in the sense of (4.3).

\section{REFERENCES}

1. S. Aizicovici, On a semilinear Volterra integrodifferential equation, Israel J. Math. 36 (1980), 273-284.

2. S. Aizicovici and N.S. Papageorgiou, Multivalued Volterra integral equations in Banach spaces, Funkcial. Ekvac., to appear.

3. P. Baras, J.C. Hassan and L. Véron, Compacité de l'opérateur définissant la solution d'une équation d'évolution non homogène, C. R. Acad. Sci. Paris, Sér. I Math., 284 (1977), 799-802.

4. R.W. Carr and K.B. Hannsgen, A nonhomogeneous integrodifferential equation in Hilbert space, SIAM J. Math. Anal. 10 (1979), 961-984.

5. - Resolvent formulas for a Volterra equation in Hilbert space, SIAM J. Math. Anal. 13 (1982), 459-483.

6. G. Da Prato and M. Iannelli, Linear integro-differential equations in Banach spaces, Rend. Sem. Math. Padova 62 (1980), 207-219.

7. A. Fryszkowski, Continuous selections for a class of non-convex multivalued maps, Studia Math. 78 (1983), 163-174.

8. K.B. Hannsgen and R.L. Wheeler, Behavior of the solution of a Volterra equation as a parameter tends to infinity, J. Integral Equations 7 (1984), 229-237.

9. T. Kiffe, A perturbation of an abstract Volterra equation, SIAM J. Math. Anal. 11 (1980), 1036-1046.

10. E. Hille and R.S. Phillips, Functional analysis and semi-groups, Amer. Math. Soc. Colloq. Pub., Vol. 31, Providence, RI, 1957.

11. N. S. Papageorgiou, Convergence theorems for Banach space valued integrable multifunctions, Intern. J. Math. Math. Sci. 10 (1987), 433-442. 
12. A. Pazy, A class of semi-linear equations of evolution, Israel J. Math. 20 (1975), 23-36.

13. - Semigroups of linear operators and applications to partial differential equations, Appl. Math. Sci., Vol. 44, Springer-Verlag, Berlin, 1983.

14. J. Prüss, Positivity and regularity of hyperbolic Volterra equations in Banach spaces, Math. Ann. 279 (1987), 317-344.

15. - Regularity and integrability of resolvents of linear Volterra equations, in Volterra integrodifferential equations in Banach spaces and applications (G. Da Prato and M. Iannelli, eds.), Pitman Res. Notes Math. Ser. 190 (1989), 339-367.

16. Linear evolutionary integral equations in Banach spaces and applications, to appear.

17. C.C. Travis and G.F. Webb, Second order differential equations in Banach spaces, in Nonlinear equations in abstract spaces (V. Lakshmikantham, ed.), Academic Press, New York, 1978, 331-361.

18. I.I. Vrabie, Compactness methods for nonlinear evolutions, Pitman Monographs Surveys Pure Appl. Math., Vol. 32, Harlow, 1987.

19. D.H. Wagner, Survey of measurable selection theorems, SIAM J. Control Optim. 15 (1977), 859-903.

Department of Mathematics, Ohio University, Athens, OH 45701-2979

Department of Mathematics and Interdisciplinary Center for Applied Mathematics, Virginia Polytechnic Institute and State University, BlacksBurg, VA 24061-0123 